DOCTRINA

\title{
Tributación en la economía digital: Propuestas impulsadas por la OCDE y el impacto frente a los principios rectores de todo sistema tributario
}

\author{
Taxation in the digital economy: Proposals promoted by the OECD \\ and the impact on the guiding principles of any tax system
}

\author{
Antonio Faúndez-Ugalde \\ Pontificia Universidad Católica de Valparaíso, Chile \\ Álvaro Vidal Olivares (1D \\ Pontificia Universidad Católica de Valparaíso, Chile
}

Alexander Olguín Romero (iD)
Universidad de Chile

Felipe Molina Marisio is

Universidad Andrés Bello, Chile

\begin{abstract}
RESUMEN El presente trabajo tiene como propósito analizar las distintas propuestas formuladas por la OCDE y el G-20 orientadas a establecer un criterio fiscal común en la economía digital desde el año 1997, en la Conferencia de Finlandia, hasta los últimos acuerdos adoptados en el año 2020, de manera de identificar los principios jurídicos que sirvieron de base a los primeros estudios y establecer cómo dichos principios han sido abordados frente a los avances en las nuevas formas de mercado digital desarrolladas en los últimos años. La recopilación de datos, la creación de valor, las plataformas de monopolio digital o los paraísos fiscales digitales son las prácticas introducidas en la economía digital que han tenido mayor desarrollo, ante lo cual se hace necesario establecer si principios jurídicos como legalidad, certeza, simplicidad, neutralidad y equidad, requieren o no considerar nuevos alcances frente a este fenómeno jurídico tributario.
\end{abstract}

PALABRAS CLAVE Tributación, economía digital, comercio electrónico, OCDE, sistema tributario. 


\begin{abstract}
The purpose of this paper is to analyze the different proposals that have formulated by the OECD and the G-20 with the goal to establish a common fiscal criterion in the digital economy since 1997, at the Finnish Conference, he until the last agreements adopted in 2020 by the entity, in order to identify the legal principles that served as the basis for the first mentioned studies and establish how these principles have been addressed over time in the face of the new forms of digital market that have been developed in recent years. Data collection, value creation, digital monopoly platforms and digital tax havens are all practices that have been introduced in the modern digital economy with a relevant development in recent years. Because of it, it is necessary to determine if the legal principles such as: legality, simplicity, neutrality, and equity have to take into consideration new scopes of application against this tax legal phenomenon.
\end{abstract}

KEYWORD Taxation, digital economic, electronic commerce, OECD, tax system.

\title{
Introducción
}

La digitalización tanto de bienes como de prestación de servicios ha planteado nuevos desafíos tanto para los operadores de dicho mercado como para la fiscalidad internacional, donde la presencia física ya no es impedimento para la expansión del comercio internacional. Las formas más conocidas de comercio electrónico o digital son aquellas desarrolladas entre empresas (business to business o $B 2 B$ ); entre empresa y consumidor (business to consumers o $B 2 C$ ); entre empresa y la administración (business to administrations o B2A); entre empresa y los empleados (business to employee o $B 2 E$ ), y entre consumidores (consumer to consumer o $C_{2} C$ ).

Estos nuevos intermediarios del mercado digital elaboran productos soportando menores costos en comparación con los agentes del comercio tradicional; se trata de empresas que, sin tener activos físicos o empleados bajo su dependencia directa dedicados a la actividad en cuestión, ocupan una posición importante en la industria donde operan (Hernández y Albagli, 2017: 2). Estos modelos desafían en particular la suposición de que una empresa no residente solo puede operar de manera significativa en una jurisdicción del mercado mediante su exclusiva presencia física (OCDE, 2015: 98). Tan trascendental y relevante ha sido el efecto que ha generado la tecnología en el mercado, que se ha denominado a esta nueva etapa «la cuarta Revolución Industrial». Este fenómeno «habría comenzado al inicio de este siglo y se caracterizaría por un internet más ubicuo y móvil, por sensores más pequeños y potentes que son cada vez más baratos, y por la inteligencia artificial y el aprendizaje de la máquina» (Schwab, 2016: 5). Hoy, en los países con alto desarrollo tecnológico tales como Japón, Estados Unidos y los de la eurozona, la economía digital es del orden de $7 \%$ al $8 \%$ del PIB, y hacia fines de esta década se prevé un 12\% (Lever, Yukich y Cruz, 2016: 6; Faúndez-Ugalde, Mellado-Silva y Blanco, 2021: 315). 
Chile no ha quedado ajeno a esta nueva realidad. La economía digital habría alcanzado ventas totales por casi US $\$ 40$ mil millones en 2015 , un $11 \%$ por sobre lo registrado el año anterior, representando alrededor del 3\% del PIB, similar al que tiene la industria alimenticia, el sector silvoagropecuario o los servicios de electricidad, gas y agua, y hacia el año 2020 su incidencia continuaría expandiéndose hasta alrededor de 4,5\% (Lever, Yukich y Cruz, 2016: 6).

Sin embargo, el fenómeno anterior se ha posicionado como uno de los principales problemas en el ámbito del derecho tributario, especialmente por la falta de adecuación normativa, generando efectos negativos en la recaudación fiscal. Así, por ejemplo, Agrawal (2017) sostiene que es evidente que si las transacciones en línea no están sujetas a impuestos, internet se puede convertir en un paraíso fiscal. Agrega que los estudios existentes indican que los individuos son sensibles a las diferencias de precios; en tal sentido, las tasas impositivas también serán sensibles a la capacidad de respuesta de las personas a las compras por internet libres de impuestos. Por su parte, Bloch y Demange (2018), y Becker y otros (2018 y 2019) analizan los modelos de negocio digitales destinados a recopilar datos que se constituyen en activos de incalculable valor y que incrementan el patrimonio de las empresas. Bourreau y otros (2018) también ilustran los problemas que pueden derivar de las denominadas plataformas de monopolio digital, que recopilan gran cantidad de datos que a su vez generan creación de valor no sometida a impuestos. En tanto, Bacache-Beauvallet (2018) plantea los problemas de competencia fiscal en el comercio electrónico, demostrando que la falta de información que se presenta en las transacciones en línea genera importantes fugas fiscales.

Los problemas anteriores derivados de estos nuevos mercados digitales no afectan solo los niveles de recaudación fiscal para contribuir en el gasto social, sino también han significado mayores costos de implementación para las administraciones fiscales y para los administrados que deben ajustarse a estos nuevos mecanismos de fiscalización electrónicos (Yilmaz y Coolidge, 2013; Banco Mundial, 2016: 17; FaúndezUgalde y otros, 2018).

Así, los antecedentes anteriores han llevado a intensos estudios desarrollados por los países de la Organización para la Cooperación y el Desarrollo Económicos (OCDE) y del Grupo de los 20 (G-20) ${ }^{1}$, los cuales presentaron en el año 2013 un plan de acción denominado Erosión de la base imponible y traslado de beneficios (siglas en inglés BEPS). «Dicho plan de acción está encaminado a garantizar que los beneficios se gravan allí donde se realizan las actividades económicas que los generan y donde se crea valor» (OCDE, 2014b: 3). Estas medidas se estructuraron con la finalidad de

1. El grupo de los 20 (G-20) está compuesto por las principales economías del mundo, esto es, la Unión Europea, Alemania, Arabia Saudita, Argentina, Australia, Brasil, Canadá, China, Corea del Sur, Estados Unidos, Francia, India, Indonesia, Italia, Japón, México, Reino Unido, Rusia, Sudáfrica y Turquía. 
otorgar armonía en la interpretación de los convenios de doble tributación internacional, considerando la gran expansión del comercio e inversiones internacionales.

La acción 1 BEPS aborda los desafíos de la economía digital para la tributación de las empresas multinacionales. Para el año 2018, se emitió un informe denominado Desafíos fiscales derivados de la digitalización: Informe provisional 2018, que identifica los negocios digitales más comunes, las dificultades frente a la legislación interna de cada país y las posturas que se han adoptado como reacción a esta tipología económica. La discusión actual se ha centrado en dos pilares de solución cuya definición aún se encuentra pendiente.

De esta manera, la recopilación de datos, la creación de valor, las plataformas de monopolio digital o los paraísos fiscales digitales, son prácticas introducidas en la economía digital que han tenido mayor desarrollo en los últimos años, siendo necesario establecer si los principios jurídicos de legalidad, certeza, simplicidad, neutralidad y equidad, requieren o no considerar nuevos alcances frente a este fenómeno jurídico tributario. Considerando lo anterior, en una primera parte se analizará el panorama internacional frente a la tributación de la economía digital, abordando los primeros esfuerzos internacionales en la búsqueda de un criterio fiscal común global, desde el año 1997 en la Conferencia de Finlandia Eliminación de barreras al comercio electrónico mundial, hasta los últimos acuerdos adoptados en el año 2020. El análisis anterior permitirá identificar los principios jurídicos que sirvieron de base a los primeros estudios y establecer cómo dichos principios han sido abordados frente a los avances de las nuevas formas de mercado digital que se han desarrollado en los últimos años. Sin duda, el mercado digital presente en los años noventa es muy distinto al desarrollado actualmente, de ahí, entonces, la necesidad de revisar el impacto en los principios jurídicos rectores de todo sistema tributario.

\section{Panorama internacional frente a la tributación de la economía digital}

Desde las primeras etapas de desarrollo de la economía digital se ha generado una serie de estudios, propuestas, conferencias y soluciones fiscales en el ámbito internacional. En el año 1997 fue celebrada en Turku, Finlandia, la conferencia titulada Eliminación de barreras al comercio electrónico mundial, instancia que reunió a representantes de gobiernos y empresas con la finalidad de debatir sobre los retos planteados por el comercio electrónico internacional a los sistemas tributarios. Según da cuenta el reporte de la OCDE (2002: 3), a menos de un año de la conferencia de Turku se concretó una nueva conferencia en Ottawa, instancia que permitió publicar uno de los instrumentos esenciales para el comercio electrónico internacional denominado Marco Tributario. Este instrumento otorgó los votos de confianza para que la OCDE coordinara los trabajos destinados a lograr un consenso internacional en esta materia. 
Estos primeros trabajos sirvieron de base para la elaboración del plan de acción contra la erosión de la base imponible y el traslado de beneficios (BEPS) del año 2013, desencadenándose, posteriormente, el primer informe del grupo de expertos sobre economía digital (GEED) de 2014; luego, el informe final del plan de acción BEPS sobre acción 1 del año 2015, y el informe provisional sobre los desafíos fiscales derivados de la digitalización del año 2018. Veamos los alcances de cada uno de ellos.

\section{La conferencia de Ottawa}

En la conferencia de Ottawa del año 1998, que llevó por título Un mundo sin fronteras - Identificación del potencial del comercio electrónico, se concluyó que los principios que regulaban el comercio convencional también debían ser aplicados al comercio electrónico (Faúndez-Ugalde, Mellado-Silva y Blanco, 2021: 319). Los principios de neutralidad, eficiencia, certeza y simplicidad, efectividad y justicia, y flexibilidad configuraron el denominado Marco Tributario, conocido también como Condiciones tributarias marco.

De acuerdo con las conclusiones y recomendaciones del Comité de Asuntos Fiscales de la OCDE (2002:3) se establecieron los alcances de cada uno de los principios que conformaron el Marco Tributario. En cuanto al principio de neutralidad, «la tributación fiscal debería tratar de ser neutral y equitativa entre las formas del comercio electrónico y entre este y el comercio convencional, evitando así la doble imposición o la no tributación involuntaria». De esta manera, la neutralidad debe tender a que las decisiones corporativas no sean motivadas por factores tributarios, lo que debe partir de la base que los estados acuerden criterios equitativos - horizontales y verticales - en la forma de gravar las operaciones en el comercio digital.

En relación a los demás principios, el reporte de la OCDE (2002: 3) plantea que en virtud de la eficiencia «se debería reducir al máximo los costos de cumplimiento para las empresas y para la administración»; si se considera la certeza y simplicidad, «las reglas fiscales deberían ser claras y fáciles de entender, de forma que los sujetos pasivos conozcan su situación», es decir, el Estado debe entregar las herramientas a los contribuyentes para anticiparse a las consecuencias fiscales, informando cuándo, dónde y cómo satisfacer sus obligaciones fiscales; por su parte, a través de la efectividad y justicia «la tributación fiscal deberá producir la cantidad correcta de impuestos en el momento adecuado, reduciendo al mínimo las posibilidades de evasión y de evitación»; y, finalmente, «los sistemas tributarios deberían ser flexibles y dinámicos para asegurar su evolución paralela a los avances tecnológicos y comerciales».

Entre las conclusiones que derivaron de la conferencia de Ottawa figura que los principios anteriores aplicados al comercio convencional pueden servir a las administraciones fiscales para regular el comercio electrónico, aunque no se excluye la generación de nuevas normas administrativas o legislativas, «siempre y cuando va- 
yan encaminadas a apoyar la aplicación de los principios de tributación fiscal y no pretendan imponer un tratamiento impositivo discriminatorio de las transacciones del comercio electrónico» (OCDE, 2002: 4). Sin embargo, en esta declaración se buscó como mecanismo de solución conciliar los fundamentos tributarios tanto para el comercio convencional como el comercio digital, situación que podría derivar en vulneración de principios como el de equidad, como se verá más adelante.

Con posterioridad a la conferencia de Ottawa, el Comité de Asuntos Fiscales de la OCDE (2002: 5) reconoció la necesidad de generar un mayor acercamiento con el sector empresarial y los países no miembros de la OCDE, pues, de lo contrario, las condiciones del Marco Tributario podrían tener problemas en su aplicación a nivel mundial. Como una medida de solución, se crearon cinco grupos consultivos técnicos (siglas en inglés TAG), formados por integrantes de las administraciones de países miembros y no miembros, y de empresas.

Los TAG se establecieron en el año 1999 con las siguientes materias (OCDE, 2002: 6): TAG de caracterización del tratado, destinado a establecer la aplicación de la definición de cánones en el contexto del comercio electrónico; TAG sobre beneficios empresariales; TAG de impuestos sobre el consumo; TAG tecnológico; y TAG de evaluación profesional de datos o de información internacionalmente compatible y requisitos de conservación de registros. Si bien los primeros resultados de los TAG fueron entregados durante los años 2000 y 2001, transcurrió más de una década para constatar los primeros indicios de un acuerdo internacional común con miras a regular la economía digital, como se analizará a continuación.

\section{El plan de acción BEPS}

Luego del descubrimiento y exposición de una serie de planificaciones fiscales llevadas a cabo por empresas multinacionales que utilizaron ventajas en la interacción de los distintos sistemas tributarios, las que implicaron una reducción de la base imponible o traslado de beneficio a países de baja tributación, a instancia de los países miembros del G-20, la OCDE publicó un plan de acción contra la erosión de la base imponible y el traslado de beneficios (BEPS) en julio de 2013.

En total, el plan de acción BEPS fue estructurado en 15 acciones. La acción 1 del proyecto apuntó a diseñar un plan de trabajo para abordar los desafíos fiscales que plantea la economía digital. Se pretendía extraer una serie de soluciones como la imposibilidad de sujetar a tributación las rentas denominadas «virtuales», para lo que también engarzaba con otras acciones no menos problemáticas como, por ejemplo, la acción 7, que lleva por título Impedir la elusión artificiosa del estatuto de establecimiento permanente, siendo, por tanto, una acción con un carácter claramente transversal (Cruz y Sánchez-Archidona, 2017: 7).

En la presentación del plan BEPS se indicó: 
El Proyecto BEPS marca un punto de inflexión en la historia de la cooperación internacional en materia de tributación. Como el marco actual basado en el consenso está en riesgo, es fundamental que se adopte una metodología adecuada que asegure que el trabajo sea inclusivo y eficaz, teniendo en cuenta la perspectiva de los países en desarrollo y los beneficios de las aportaciones de los negocios y la sociedad civil en general (OCDE, 2014a: 30).

Siguiendo la descripción de la acción 1, la tarea encomendada consistió en «identificar las principales dificultades que planteaba la economía digital, para la aplicación de las normas de fiscalidad internacional y desarrollar opciones detalladas para abordar estas dificultades, con un enfoque holístico y considerando tanto la imposición directa como la indirecta» (OCDE, 2014a: 33). De acuerdo a lo anterior, el reporte de la OCDE definió temas y situaciones a examinar, entre los que figuran la capacidad de una compañía para tener una presencia digital significativa en la economía de otro país sin estar sujeta a imposición — debido a la falta de un nexo bajo las normas internacionales actuales-; la atribución del valor creado a partir de la generación de datos comercializables de ubicación relevante mediante el uso de productos y servicios digitales; la caracterización de los ingresos procedentes de los nuevos modelos comerciales; y la aplicación de normas relativas a la tributación en fuente y el modo de asegurar la recaudación efectiva del impuesto al valor agregado, como también de otros impuestos indirectos relativos al suministro transfronterizo de bienes y servicios digitales (OCDE, 2014a:33).

\section{El informe del GEFED}

Dos meses después de la publicación del Plan BEPS, se creó el Grupo de Expertos sobre Fiscalidad de la Economía Digital (GEFED), con un carácter auxiliar al comité de asuntos fiscales de la OCDE, destinado a emitir informes con los problemas detectados por la economía digital y proponer posibles soluciones. Dicho informe, presentado en el año 2014, tuvo como fuente una amplia y transversal consulta a representantes empresariales, sindicatos, organizaciones de la sociedad civil, y miembros del sector académico, pertenecientes tanto a estados miembros como a países en desarrollo y otras economías no pertenecientes a la OCDE.

La OCDE (2014b: 31) informó que el GEFED consideró que los «principios [declarados en la conferencia de Ottawa] siguen siendo un importante referente hoy día $y$, reforzados en su caso, pueden constituir la base para valorar las posibles opciones con miras a afrontar los desafíos fiscales de la economía digital». En este sentido, aún resulta imprescindible, frente a los nuevos desafíos de la economía digital, considerar para un sistema tributario los principios de neutralidad, eficiencia, certeza y simplicidad, efectividad y justicia, y la flexibilidad y sostenibilidad. De acuerdo con la OCDE (2014b: 162), construir este marco normativo fiscal, garantizaría «un análisis 
coherente y objetivo, a cuyo fin no podrá prevalecer ningún principio sobre los restantes»; por el contrario, la referida evaluación deberá apoyarse en un examen global de los distintos factores que conforman dicho marco tributario. Sin embargo, bajo el criterio del Marco Tributario de Ottawa tendiente a otorgar el mismo tratamiento normativo tanto para el comercio convencional como el digital, seguía siendo un contrasentido frente a los principios tributarios recogidos por el GEFED.

El capítulo 5 del informe del GEFED efectuó una descripción detallada de los elementos esenciales típicos de las prácticas elusivas BEPS en el contexto de la economía digital. Para tal efecto, se clasificaron las prácticas en dos grupos relacionados con la imposición directa e indirecta. En el primer grupo, el informe contempla cuatro categorías de prácticas (OCDE, 2014a: 112): 1) La minimización de la carga tributaria en el Estado de la fuente evitando toda presencia fiscalmente imponible o, en caso de tener una presencia fiscalmente imponible, traslado de los beneficios brutos mediante estructuras comerciales o reducción del beneficio neto, maximizando las deducciones a nivel del ordenante; 2) La escasa o nula retención en la fuente; 3) La escasa o nula tributación a nivel del perceptor de las rentas; y 4) La no imposición de los rendimientos sometidos a baja tributación a nivel de la matriz.

En el segundo grupo referente a prácticas BEPS en el marco de impuestos indirectos, el informe los circunscribe (OCDE, 2014a: 118) a los suministros digitales a distancia a empresas exentas; $y$ a los suministros digitales a distancia a empresas con establecimientos en más de una jurisdicción que se dedican a actividades exentas.

Las soluciones propuestas por los dos grupos se encaminaron a fijar un nuevo nexo con base en una presencia virtual significativa, sustituyendo de esta forma el concepto existente de establecimiento permanente; someter a las transacciones digitales a una retención en la fuente; introducir un impuesto sobre las transacciones electrónicas o bit tax; la revisión de exenciones de mercaderías de escaso valor; y el registro de todo proveedor residente en la jurisdicción del consumidor. El informe culmina estableciendo que todas las soluciones planteadas deben ser exhaustivamente revisadas, para lo cual se fijó como plazo de término de la investigación el año 2015.

\section{El informe final del plan BEPS}

El reporte de la OCDE (2015) sobre los desafíos fiscales relacionados con la acción 1, deja de manifiesto los problemas que hasta esa fecha se presentaban a nivel de impuestos directos, situación que, como se verá más adelante, aún espera un consenso internacional.

Por un lado, el documento acepta introducir modificaciones relacionadas a los alcances del concepto de establecimiento permanente en cuanto a su naturaleza preparatoria o auxiliar. Por otro lado, sin embargo, el reporte (OCDE, 2015: 13) rechaza las recomendaciones realizadas por el GEFED en cuanto a un nuevo nexo 
sobre la presencia económica significativa, la retención en origen sobre ciertos tipos de transacciones digitales, y a establecer una tasa de compensación. El reporte (OCDE, 2015: 13) indica que dicho rechazo se debe «entre otras razones, a que se espera que las medidas desarrolladas en el Proyecto BEPS tengan un impacto sustancial en los problemas BEPS previamente identificados en la economía digital, que ciertas medidas BEPS mitigarán algunos aspectos de los desafíos tributarios más amplios, y que los impuestos sobre el consumo se aplicarán eficazmente en el país del mercado».

Como medidas futuras, el mismo documento plantea que sus conclusiones pueden evolucionar a medida que la economía digital continúa desarrollándose, frente a lo cual es importante seguir trabajando en estos temas y monitorear los desarrollos a lo largo del tiempo, sugiriendo elaborar un nuevo informe para el año 2020.

\section{El informe provisional del año 2018}

Si bien el informe del plan BEPS del año 2015 sugirió un nuevo informe para el año 2020, en marzo de 2017 los ministros de finanzas del G-20 encomendaron a la OCDE, bajo los auspicios del denominado Marco Inclusivo sobre BEPS, la elaboración de un informe provisional para el año 2018. Dicho informe provisional fue publicado en marzo de 2018, permitiendo constatar la presencia de dos grupos de países que comenzaban a establecer dos líneas argumentativas sobre la forma de tributación en la economía digital, alejando una posible unificación de criterios.

De acuerdo con el resumen del informe (OCDE, 2018) se presentan las siguientes posturas: aquellos países que opinan que no se requiere acción alguna, aquellos que consideran necesario adoptar medidas que tengan en cuenta las contribuciones de los usuarios, y aquellos países que sostienen que todo cambio o modificación acometidos han de aplicarse con carácter generalizado a toda actividad económica.

En esta etapa se puede apreciar la intención de los países miembros de establecer los alcances del «reparto de la potestad tributaria entre jurisdicciones y a la forma en que se asignan los beneficios a las diferentes actividades desarrolladas por las empresas multinacionales» (OCDE, 2018: 5), para lo cual proponen seguir los intentos en definir, por un lado, un criterio de determinación en función de un nexo o criterio de sujeción, y, por otro, reglas de atribución de beneficios.

Sin perjuicio de lo anterior, el informe promueve por parte de los países llevar a cabo medidas provisionales en forma unilateral, entre las cuales se encuentra el establecimiento de un impuesto especial a ciertos servicios digitales. Sin embargo, el informe deja constancia de los países opositores a tales medidas bajo el fundamento que «generarán riesgos y tendrán efectos adversos como pueden ser repercusiones negativas en la inversión, la innovación y el crecimiento; una posible sobreimposición; efectos distorsionadores de la producción; el aumento de la carga fiscal sopor- 
tada por consumidores y empresas y unos costes de cumplimiento y gastos administrativos más elevados, entre otros» (OCDE, 2018: 5).

Así, comenzaron a tener espacio los primeros impuestos a los servicios digitales como medidas a corto plazo como ocurrió en la Unión Europea (COM, 2018b: 40) y, también, en algunos países en Latinoamérica como Argentina, Colombia y Uruguay (Carbajo, 2019). Si bien estas medidas provisionales han seguido avanzando en otros países, el acuerdo común internacional aún sigue pendiente hasta este período, sembrando nuevas dudas, especialmente en cómo el derecho tributario internacional va tomando partido en la soberanía de los estados. Siendo así, las iniciativas unilaterales de los países en la fiscalidad internacional se han adelantado a las medidas multilaterales, aunque como plantean Báez y Brauner (2019: 16), técnicamente la solución multilateral es bastante similar a la solución unilateral; la única diferencia está en la estandarización del mecanismo para la adopción de dicha legislación, en donde, naturalmente la adopción multilateral puede seguir a la adopción unilateral iniciada por varios países.

\section{La policy note del año 2019 y últimos trabajos del 2020}

Como se analizó en el acápite anterior, el informe provisional del año 2018 solamente desarrolló las diferencias que existían entre los países miembros y promovió la aplicación, a corto plazo, de medidas unilaterales como el impuesto a los servicios digitales. Así, en enero de 2019, la OCDE (2019a) emitió una nota guía para someter a consulta pública propuestas concretas en las que describía cada uno de los pilares en los cuales se centraba la discusión sobre la tributación en la economía digital. La publicación llevó por título Addressing the tax challenges of the digitalization of the economy.

Estas propuestas parecen provenir principalmente del grupo de estados cuyas jurisdicciones han resultado más desfavorecidas a causa de la baja tributación por parte de empresas altamente digitalizadas, en desmedro no solo de los estados afectados, sino también respecto a empresas que desarrollan el comercio convencional o tradicional (Faúndez-Ugalde, Mellado-Silva y Blanco, 2021: 321).

El comunicado de la OCDE establece que las propuestas se basan en dos pilares (OCDE, 2019b). El pilar I presentó tres propuestas: la participación del usuario (user participation); los intangibles de comercialización (marketing intangibles), y la presencia económica significativa (significant economic presence). Actualmente, la OCDE ha unificado el primer pilar en el sentido de que sea aplicado a sujetos pasivos determinados, permitiendo gravar los resultados de la actividad digital. La dificultad se puede presentar en la forma de cálculo de las ganancias residuales que se generen en una jurisdicción de mercado y, una vez determinada, se tendría que definir la forma de reparto de dicha ganancia residual entre los mercados participantes (FaúndezUgalde, Mellado-Silva y Blanco, 2021: 321). 
El pilar II se sustenta en los riesgos BEPS remanentes, buscando establecer una tributación mínima o negando beneficios de los tratados si el beneficiario está sujeto a nula o baja tributación. Esta propuesta tenía como principal sustento la "propuesta global contra la erosión de las bases imponibles» (Global Anti-Base Erosion o Glo$B e$ ), que derivan de las medidas antielusivas estadounidenses del año 2017, esto es, el Global Intangible Low Tax Income (GILTI) y el Base Erosion Anti-abuse Tax (BEAT). Según Ortega (2019), estas últimas reformas fiscales de Estados Unidos estaban destinadas a reforzar la capacidad impositiva de los países en aquellos casos en los que la otra jurisdicción aplique tipos impositivos reducidos sobre los beneficios en cuestión.

Los trabajos de la OCDE (2019c) realizados hasta fines de 2019 se centraron en definir una evaluación del impacto económico de las propuestas, y hasta octubre de 2020 se incluye una nueva versión de los pilares con los correspondientes estudios económicos (OCDE, 2020a y 2020b).

\section{Nuevas formas de comercio digital y su impacto en los principios jurídicos tributarios}

Ha quedado de manifiesto que el establecimiento de los dos pilares de la OCDE, en sí, presentan un importante grado de complejidad para la tributación en la economía digital. El comercio internacional revela que los negocios son altamente digitalizados y las propuestas fiscales que derivan de los grupos de trabajo de la OCDE abren una serie de interrogantes frente a los servicios de redes sociales, análisis de datos y plataformas digitales. A esto se suman las medidas unilaterales decretadas por los estados restando toda posibilidad de establecer un criterio internacional común de tributación o, como plantean Báez y Brauner (2019: 7), socavan el régimen fiscal internacional existente y sus logros no triviales hasta la fecha, retratando una imagen poco realista de la temporalidad y delimitando la economía digital o partes de ella sin abordar las cuestiones clave de BEPS.

Así, por ejemplo, si nos remitimos al pilar I, se presenta la propuesta de participación del usuario, donde cobra relevancia en sus operaciones la recopilación de datos, la creación de valor, las plataformas de monopolio digital, y los paraísos fiscales digitales, entre otros. Estas son prácticas introducidas en la economía digital que han tenido el mayor desarrollo en los últimos años y ante las que -independiente de la solución final que se pueda consensuar, multilateral o unilateralmente- no se pueden perder de vista los principios que reconoce el derecho tributario internacional, especialmente aquellos declarados en la conferencia de Ottawa del año 1998. Así, la economía digital como parte del fenómeno jurídico tributario ${ }^{2}$ requiere el estudio

2. En cuanto al fenómeno jurídico, Squella (2007) señala que el derecho es un fenómeno preferentemente normativo porque en él hay también otros estándares, distintos de las normas, tales como los 
de los principios tributarios para enfrentarlos a estas nuevas formas de negocios digitalizados y que forman parte de los pilares de la OCDE actualmente en discusión.

Particularmente en relación con el fenómeno jurídico tributario, el derecho necesita considerar el dinamismo de las formas de contratación que se presentan en la economía digital, solo así se puede establecer un análisis sistematizado y coherente entre las normas, principios y hechos que derivan del ámbito digital. Si bien el fenómeno jurídico se inicia con el establecimiento de normas jurídicas, estas son una parte de dicho fenómeno, siendo indispensables otras tres dimensiones que le otorga un fidedigno observador: el derecho vivido que surge de los hechos, la jurisprudencia, y la doctrina (Vergara, 2015). Son estas cuatro dimensiones las que permitirán una mejor comprensión de las nuevas formas de contratación en la economía digital y el impacto en los principios jurídicos, como se verá a continuación.

\section{Recopilación de datos y creación de valor}

Actualmente se pueden presentar determinados servicios o plataformas que, por particularidades de la más variada índole, no estén en condiciones de gravar en algunas jurisdicciones, y que podrían quedar afectas a una carga tributaria diversa o bajo distintas condiciones a aquellas que se aplican a otro tipo de servicios o plataformas, generando además problemas de localización frente a procesos de fiscalización por parte de las administraciones fiscales. La situación anterior es de vital importancia, pues si las transacciones en línea no están sujetas a impuestos, internet se puede convertir en un paraíso fiscal (Agrawal, 2017).

El denominado proceso del data mining se ha constituido en uno de los desarrollos de conocimiento más relevantes en la recopilación, transformación y uso de datos entre los modelos de negocio. Uno de los casos más relevantes fue Facebook, cuyo cofundador y director ejecutivo, Mark Zuckerberg, reconoció en el año 2018 que se habría generado un uso indebido de datos de 87 millones de usuarios por parte de Cambridge Analytica durante la campaña de las elecciones presidenciales del año 2016 en Estados Unidos. De esta forma, la recopilación de datos de los usuarios o el registro de su historial de actividad en la plataforma digital puede dar lugar a posibles ingresos por venta de datos personales (Bloch y Demange, 2018: 4).

Bloch y Demange (2018) plantean dos cuestionamientos frente a la recopilación

\footnotetext{
principios y los valores. Por su parte, Goldschmidt (1962) plantea elementos de diferente índole en el fenómeno jurídico: primero, conductas llevadas a cabo por jueces, secretarios, funcionarios, abogados; segundo, nos conmueven estas conductas, sea que su justicia nos complazca y reconforte, sea que su injusticia nos indigne y subleve; y tercero, nos enteramos de lo jurídico cuando estudiamos los códigos del país y el sinfín de manuales y tratados dedicados a su análisis. Por lo tanto, para efectos de nuestro estudio, el fenómeno será normativo porque puede ser considerado con base en la experiencia jurídica, y tributario, por su vinculación con las normas jurídicas tributarias.
} 
de datos. Lo primero es que los datos son una entrada valiosa para las plataformas de internet, pero los usuarios voluntariamente suben sus datos sin ningún pago. Así, las plataformas de internet podrían estar involucradas en un acuerdo de trueque, donde brindan un servicio valioso a cambio de la carga de datos. Lo segundo es que los usuarios temen con razón que la recopilación de datos personales infrinja su privacidad; es decir, si bien la explotación de datos proporciona un servicio valioso para los usuarios al mejorar la orientación, también implica necesariamente un costo en la pérdida de privacidad.

En estos nuevos modelos de negocio resulta relevante la publicidad, pues las empresas explotan datos a una escala mucho mayor que en el pasado. Tanto la creación como el mantenimiento de una red de fuentes de datos, que se recopilan de manera sistemática y sostenida a gran escala, constituyen un importante factor de «creación de valor» para muchos modelos de negocio en la economía digitalizada (Becker y otros, 2018). Sin embargo, las distintas propuestas que los países han planteado a nivel OCDE dejan en evidencia la falta de acuerdo en establecer los factores que inciden en la creación de valor. El Informe Final de la Acción 1 (OCDE, 2015) sugiere que el valor de los datos se deduzca de los ingresos que reporten los servicios de publicidad. En cambio, el Informe Provisional (OCDE, 2018) no hace ninguna referencia sobre la forma de determinación del valor de los datos.

La realidad de hoy demuestra cómo las grandes plataformas de ventas utilizan registros históricos de ventas para dar intención a una publicidad directa a usuarios específicos. Por lo tanto, estos nuevos modelos de negocio dependen de la recopilación de datos personales, constituyéndose en un activo de incalculable valor que puede pasar desapercibido frente a las administraciones fiscales.

Tratando de buscar un correcto alcance sobre la creación de valor, Becker y otros (2019) indican que hay que considerar las relaciones jurídicas que se presentan entre las empresas y los usuarios, lo que denominan «relaciones sostenidas con los usuarios» (siglas en inglés SURE), destacando aspectos económicos y legales vinculados a las reglas del nexo y a las de asignación de beneficios. Es precisamente en este tipo de relaciones en el ámbito de la economía digital donde se identifican operaciones de explotación de datos, de publicidad y de servicios de plataforma. Los mismos autores destacan que es la firma en sí, no el cliente, lo que crea valor, y que esta creación de valor puede tener lugar en la ubicación del cliente porque, como tal, una empresa digital puede participar en una actividad productiva en una jurisdicción sin estar físicamente presente allí (Becker y otros, 2018).

Otros autores como Turina (2020: 5), destacan dentro del fenómeno jurídico tributario aspectos de la teoría económica para la cuantificación de la creación de valor, señalando que, bajo criterios propios de la tributación internacional, la creación de valor se posiciona exclusivamente al «lado de la oferta»; sin embargo, frente a una comprensión más económica de la misma, requeriría implícitamente que el factor de 
la «demanda» también se tenga en cuenta, ya que, de acuerdo con la teoría subjetiva del valor dominante, la oferta no puede crear valor de forma completamente independiente de demanda.

Por su parte, la Comisión Europea (COM, 2018a), tratando de buscar una solución provisional, instruye un impuesto a los servicios digitales (ISD) que «se centra en actividades en las que existe una gran brecha entre el valor creado y la capacidad de los Estados miembros para gravarlo, donde la participación y la contribución del usuario juegan un papel central en la creación de valor». Sin embargo, Ludwig y otros (2019: 35) indican que la Comisión Europea proporciona una descripción más detallada de la brecha entre la creación de valor y la capacidad de gravar las ganancias y, en específico, no se explica por qué esta brecha es particularmente grande con respecto a las empresas que ofrecen servicios que están bajo el alcance del ISD en comparación con los servicios que están excluidos del mismo.

Por lo tanto, los factores que inciden en la creación de valor de los datos aún resul$\tan$ insuficientes, lo que puede arrastrar incongruencias frente al principio de legalidad de los tributos, especialmente en la forma de establecer las bases tributables y que dependerá, en gran medida, del consenso internacional que los países puedan lograr. Siendo así, las propuestas que se concreten al alero de la OCDE, mientras no exista un criterio uniforme internacionalmente aceptado, pueden impactar en los cuerpos normativos internos de cada país generando problemas de interpretación jurídica, especialmente en lo que se refiere al impuesto corporativo. Esto también incide en el principio de certeza y simplicidad que se ha promovido desde la conferencia de Ottawa de 1998, en el sentido de que las normas tributarias deben entregar con claridad cada uno de los elementos de las obligaciones que implican, de manera que los contribuyentes tengan el legítimo derecho de anticiparse a las consecuencias fiscales, de manera de saber cuándo, dónde y cómo dar cumplimiento a dichas obligaciones.

Algunos autores como Bloch y Demange (2018), han planteado como posible solución establecer un impuesto diferenciado que grave los ingresos de acceso y los ingresos de datos a diferentes tasas, lo que sería un instrumento efectivo. Otros como Collin y Colin (2013) indican que los datos personales son una forma de «trabajo gratuito» para plataformas en línea que deben ser gravados no solo para recaudar ingresos fiscales adicionales sino también para poner en pie de igualdad a las industrias en online y offline, lo que, además, estaría en directa relación con el principio de efectividad y de justicia en el sentido de establecer tributos idóneos en tiempo y forma, reduciendo al máximo las posibilidades de evasión y fraude fiscal.

\section{Plataformas digitales y monopolística}

Como consecuencia de la falta de un criterio internacional uniforme frente a los factores que inciden en la creación de valor en la recopilación de datos, las empre- 
sas multinacionales a través de sus plataformas digitales se pueden constituir en verdaderas plataformas monopolísticas aisladas de tributación en los países donde tienen presencia significativa. Dichas plataformas digitales monopolísticas o dominantes se caracterizan porque desarrollan un modelo basado en datos personales de los usuarios para proporcionar servicios personalizados y vender espacios publicitarios dirigidos a vendedores online (Bourreau y otros, 2018: 2). Así ha ocurrido, por ejemplo, con países de la Unión Europea donde firmas destacadas como Netflix, Spotify, Soundcloud y Amazon (con sus respectivos servicios de transmisión de video y música) no enfrentan ISD (Sheppard, 2018). Ludwig y otros (2019: 36) señalan que la aplicación de ISD únicamente a empresas seleccionadas arbitrariamente que participan en la publicidad en línea y a aquellas que tienen interfaces multifacéticas muy destacadas, no solo podría aislar lo digital de la economía tradicional, sino que incluso puede causar más barreras dentro de la propia economía digital.

Esta distorsión, ¿acaso podría afectar el principio de neutralidad? Como se declaró en la conferencia de Ottawa del año 1998, de acuerdo con el principio de neutralidad, los sistemas tributarios deben tratar de ser neutrales y equitativos entre las distintas formas de comercio electrónico y entre este y el comercio convencional o tradicional. Faúndez-Ugalde (2018: 170) plantea que la posibilidad de tratos diferenciados entre operaciones de comercio convencional de aquellas que se realizan en una economía digital debe ser entendida desde una perspectiva destinada a cumplir con el principio de neutralidad, evitando distorsiones económicas, formulando sistemas más simples y minimizando las oportunidades de elusión. De esta manera, cumplir con el principio de neutralidad no implica que necesariamente deba existir igualdad en la carga impositiva. En este sentido, el tratamiento tributario asimétrico en las operaciones de la economía digital debe estar orientado a evitar distorsiones entre los distintos agentes económicos.

Asimismo, considerando el nexo en la atribución de utilidades planteado en la policy note de la OCDE de enero de 2019, no habría obstáculo para crear tasas diferenciadas de acuerdo con los tipos de servicios o plataformas digitales que se clasifiquen. Sin embargo, el problema se ha presentado en que algunas jurisdicciones, como la chilena, se han anticipado al acuerdo unánime internacional, introduciendo regulaciones unilaterales con relación a las plataformas de internet, sometiendo dichas operaciones a un gravamen indirecto como es el impuesto al valor agregado. Esta situación puede llevar a distorsiones en el mercado interno al generar operaciones desde terceros países o triangulaciones. De ahí la necesidad de concretar un criterio internacional uniforme que tienda a la neutralidad de las decisiones corporativas. En el caso de la Comisión Europea (COM, 2017: 11), tratando de buscar una solución frente a las medidas unilaterales, en su reciente comunicación sobre un sistema fiscal justo y eficiente para el mercado único digital, se declaró lo siguiente: «Desde el inicio de su mandato, esta Comisión ha tomado medidas para garantizar el principio de que 
todas las empresas que operan en la UE deben pagar sus impuestos donde se generan beneficios y valor. Este principio es fundamental para una fiscalidad justa y eficaz en el mercado único, y solo puede aplicarse mediante medidas comunes y coordinadas».

\section{Impacto en los costos de inversión de las plataformas de internet}

Generalmente los costos de implementación de modelos de negocio a partir de plataformas digitales suelen ser bajos, con depreciaciones favorables a los software $y$, muchas veces, con incentivos tributarios de investigación y desarrollo (I+D). Esta situación favorece en la disminución de las bases imponibles y pone a este tipo de empresas en una situación fiscal mas ventajosa en comparación con aquellas que realizan comercio offline.

Ludwig y otros (2019: 34) señalan que algunas empresas que desarrollan negocios digitales realmente pagan menos impuestos de lo que se supone deben pagar, lo que puede ser el resultado de estrategias de planificación fiscal agresiva (elusión). Sin embargo, un buen sistema tributario debe generar una recaudación equitativa, con beneficios tributarios que no afecten el principio de igualdad ante la ley. En este sentido, si bien los incentivos tributarios - como el caso del I+D - tienen un sustento de política tributaria para favorecer a cierto sector de la economía o el desarrollo tecnológico del Estado, necesariamente deben considerar la situación fiscal de las empresas que desarrollan negocios digitales, especialmente si en sus operaciones en otros países no están quedando gravadas con impuestos.

\section{Conclusiones}

El estudio de las distintas propuestas de la OCDE y el G-20 relacionadas con la fiscalidad en la economía digital, desde el año 1997 en adelante, ha permitido identificar los principios jurídicos que han servido de base para los acuerdos adoptados en el seno de dichas entidades, aunque el consenso para la aplicación de impuestos directos aún es un tema pendiente de cara a la doble tributación internacional. En esta línea, la investigación da cuenta del impacto de dichos principios jurídicos frente a la evolución de los nuevos modelos de negocio digitales vinculados con la recopilación de datos, la creación de valor, las plataformas de monopolio digital y los paraísos fiscales digitales.

Como precisaron Bloch y Demange (2018), la recopilación de datos es una entrada valiosa para las plataformas de internet, pero los usuarios voluntariamente suben sus datos sin ningún pago. Así, en los nuevos modelos de negocio la explotación de datos es a una escala mucho mayor que en el pasado, constituyéndose en un importante factor de "creación de valor» que se puede pasar por alto en las administraciones fiscales. Sin embargo, la falta de precisión en los elementos que inciden en la 
creación de valor puede generar incongruencias frente al principio de legalidad de los tributos, afectando con ello la certeza y simplicidad que se ha promovido desde la conferencia de Ottawa de 1998.

Por otro lado, las plataformas digitales monopolísticas generan verdaderas barreras dentro de la propia economía digital, afectando con ello la igualdad en la industria. Si bien la conferencia de Ottawa de 1998 promueve la neutralidad y equidad entre las distintas formas de comercio electrónico, y entre este y el comercio convencional o tradicional, concluimos, sin embargo, que frente a las nuevas formas de negocio digital que operan el día de hoy, cumplir con el principio de neutralidad no implica que necesariamente deba existir igualdad en la carga impositiva. Siendo así, el tratamiento tributario asimétrico en las operaciones de la economía digital resulta indispensable para evitar distorsiones entre los distintos agentes económicos.

Finalmente, es importante rescatar que los incentivos tributarios que pueden derivar de la política tributaria que implementen los países en forma unilateral, también pueden generar efectos adversos frente a la fiscalidad en la economía digital, más aún en los casos en que las operaciones de un contribuyente no están siendo gravadas con tributos en el otro país en el que están teniendo presencia significativa.

\section{Referencias}

Agrawal, David (2017). «The Internet as a tax haven? The effect of the Internet on tax competition». DOI: 10.2139/ssrn.2328479.

Bacache-Beauvallet, Maya (2018). «Tax competition, tax coordination, and ecommerce». Journal of Public Economic Theory, 20 (1): 100-117. DOI: 10.1111/ jpet.12254.

BÁEz, Andrés y Yariv Brauner (2019). «Taxing the digital economy post BEPS... Seriously». University of Florida Levin College of Law Legal Studies Research Paper 19-16: 1-6o. Disponible en: https://bit.ly/34XthU3

Banco Mundial (2016). WORLD DEVELOPMENT REPORT 2016: Digital DiVIDENDS. WASHINGTON DC: WORLD BANK.

BeCKer, Johannes, Joachim Englisch y Deborah Schanz (2018). «How data should (not) be taxed». Disponible en SSRN: https://ssrn.com/abstract=3289036.

Becker, Johannes, Joachim Englisch y Deborah Schanz (2019). «Re-allocation of taxing rights for big data business models». Disponible en https://bit.ly/2Tw8FzL.

BLOCH, Francis y Gabrielle Demange (2018). «Taxation and privacy protection on Internet platforms». Journal of Public Economic Theory, 20 (1): 52-66. DOI: 10.1111/ jpet.12243.

Bourreau, Marc, Bernard Caillaud y Romain De Nijs (2018). «Taxation of a digital monopoly platform». Journal of Public Economic Theory, 20 (1): 40-51. DOI: 10.1111/jpet.12255. 
Carbajo, Domingo (2019).«De la tributación de la economía digital a BEPS 2.0 ¿Una nueva etapa de la fiscalidad internacional?». Revista de Fiscalidad Internacional y Negocios Transnacionales, 12: 41-64.

Collin, Pierre y Nicolas Colin (2013). Mission d'expertise sur la fiscalité de léconomie numérique. Rapport au Ministre de l'économie et des finances. Disponible en: https://bit.ly/2TuLN3E.

COM, Comisión Europea (2017). A fair and efficient tax system in the European Union for the digital single market. COM(2017) 547 final (21 septiembre 2017). Disponible en https://bit.ly/3p7EtXd.

-. (2018a). Communication from the Commission to the European Parliament and the Council. Time to establish a modern, fair and efficient taxation standard for the digital economy. $\operatorname{COM(2018)} 146$ final (21 marzo 2018). Disponible en https://bit. ly/3gloBf

-. (2018b). Propuesta de Directiva del Consejo relativa al sistema común del impuesto sobre los servicios digitales que grava los ingresos procedentes de la prestación de determinados servicios digitales. COM(2018) 148 final (21 marzo 2018). Disponible en https://bit.ly/3cubNma

Cruz, Ignacio y Guillermo Sánchez-Archidona (2017). «Economía digital, establecimiento permanente y presencia digital significativa: Tras las conclusiones del informe del GEFED». Quincena Fiscal, 18: 59-88.

FaúndeZ-Ugalde, Antonio (2018). «El problema del concepto actual de establecimiento permanente en los convenios de doble tributación internacional frente a los nuevos desafíos fiscales en la economía digital». Revista Chilena de Derecho y Tecnología, 7 (1): 155-173. DOI: 10.5354/0719-2584.2018.48223.

Faúndez-Ugalde, Antonio, Rachid Osman-Hein y Mario Pino Moya (2018). «La auditoría tributaria por sistemas electrónicos frente a los derechos de los contribuyentes: Un estudio comparado en América Latina». Revista Chilena de Derecho $y$ Tecnología, 7 (2): 113-135. DOI: 10.5354/0719-2584.2018.51099.

FaúndeZ-Ugalde, Antonio, Rafael Mellado-Silva y María Blanco (2018). «Estados Unidos versus China frente a los desafíos fiscales de la OCDE y el G-20 en el e-commerce». En Clayton Moreira da Silva (editor), Administração, finanças e geração de valor (pp. 315-325). Paraná: Atenea. Disponible en: https://bit.ly/3i6NE91.

GoldschmidT, Werner (1962). Introducción al derecho. Buenos Aires: Aguilar.

Hernández, Leonardo y Pinjas Albagli (2017). Economía digital: Oportunidades y desafíos. Santiago: Clapes UC.

Lever, George, Yerka Yukich y María Cruz (2016). La economía digital en Chile 2016. Santiago: Centro de Estudios de la Economía Digital, Cámara de Comercio de Santiago.

Ludwig, Christopher, Marcel Olbert y Christoph Spengel (2019). «Transfer pricing 
for data businesses: How to apply the arm's length principle to the digital economy». En Pasquale Pistone y Dennis Weber (editores), Taxing the digital economy (pp. 27-47). Ámsterdam: IBFD.

OCDE, Organización para la Cooperación y el Desarrollo Económicos (2002). Taxation and electronic commerce, implementation of the Ottawa taxation framework conditions. París: OECD Publishing. Disponible en: https://bit.ly/3uxfb68.

-. (2014a). Plan de acción contra la erosión de la base imponible y el traslado de beneficios. DOI:https://10.1787/9789264207813-es.

-. (2014b). Cómo abordar los desafíos fiscales de la economía digital. París: OECD Publishing.

-. (2015). Addressing the tax challenges of the digital economy: Action 1, 2015 final report. París: OECD Publishing. DOI: https://10.1787/9789264241046-en.

-. (2018). Resumen de los desafíos fiscales derivados de la digitalización: Informe provisional 2018. OECD Publishing. Disponible en https://bit.ly/2RQGhrL.

-. (2019a). Addressing the tax challenges of the digitalisation of the economy-policy note. As approved by the Inclusive Framework on BEPS on 23 January 2019. Disponible en https://bit.ly/3pzXVfr.

-. (2019b). Public consultation document, addressing the tax challenges of the digitalisation of the economy. OECD/ G2o Base Erosion and Profit Shifting Project, Public Consultation Document, 13 Februar - 6 March 2019. Disponible en https:// bit.ly/3gcTPpu.

-. (2019c). Programme of work to develop a consensus solution to the tax challenges arising from the digitalisation of the economy. OECD/G2o Inclusive Framework on BEPS. París: OECD Publishing.

-. (2020a). Tax Challenges Arising from Digitalisation - Report on Pillar One Blueprint. Disponible en https://bit.ly/3gibsEq.

-. (2020b). Tax Challenges Arising from Digitalisation - Report on Pillar Two Blueprint. Disponible en https://bit.ly/3v3rqYg.

Ortega, Mario (2019). «Economía digital: La OCDE plantea tributar allí donde se crea el valor o aplicar un impuesto mínimo mundial». Expansión. Disponible en: https://bit.ly/3g29nw4.

Schwab, Klaus (2016). La cuarta Revolución Industrial. Barcelona: Debate.

SHEPPARD, Lee (2018). «Digital permanent establishment and digital equalization taxes». Bulletin for International Taxations, 4 (special issue): 9-16.

SQuella, Agustín (2007). ¿Qué es el derecho? Santiago: Editorial Jurídica de Chile.

Turina, Alessandro (2020). "The progressive policy shift in the debate on the international tax challenges of the digital economy: A "pretext" for overhaul of the international tax regime?». Computer Law \& Security Review, 36: 1-17. DOI: 10.1016/j.clsr.2019.105382. 
Vergara, Alejandro (2015). «Delimitar y distinguir: Teoría del derecho, filosofía del derecho y doctrina jurídica». Revista de Derecho de la Pontificia Universidad Católica de Valparaíso, 44: 623-66o.

YIlmaz, Fatih y Jacqueline Coolidge (2013). «Can e-filing reduce tax compliance costs in developing countries?». Policy Research Working Paper, 6.647: 1-57. DOI: 10.1596/1813-9450-6647.

\section{Sobre los autores}

Antonio Faúndez-Ugalde es profesor de derecho tributario de la Escuela de Derecho y de la Escuela de Comercio de la Pontificia Universidad Católica de Valparaíso, Doctor en Derecho de la Pontificia Universidad Católica de Valparaíso, Magíster en Gestión mención Tributación Internacional de la Pontificia Universidad Católica de Valparaíso. Su correo electrónico es antonio.faundez@pucv.cl. (DD http://orcid. org/0000-0002-8468-3042.

Álvaro Vidal Olivares es profesor titular de derecho civil de la Escuela de Derecho de la Pontificia Universidad Católica de Valparaíso, Doctor en Derecho de la Universidad Autónoma de Madrid. Su correo electrónico es alvaro.vidal@pucv.cl. (D) https://orcid.org/oooo-0001-5362-9310.

Alexander Olguín Romero es profesor de derecho tributario en la Facultad de Economía y Negocios de la Universidad de Chile, Magíster en Tributación de la Universidad de Chile. Su correo electrónico es aolguin@fen.uchile.cl. (D) https://orcid. org/0000-0001-7610-0305.

Felipe Molina Marisio es profesor de derecho tributario en la Universidad Andrés Bello, Magíster en Tributación de la Universidad de Chile. Su correo electrónico es felipe.molina@pratmolina.cl. (iD https://orcid.org/oooo-0002-1667-3846. 


\title{
REVISTA CHILENA DE DERECHO Y TECNOLOGÍA
}

La Revista de Chilena de Derecho y Tecnología es una publicación académica semestral del Centro de Estudios en Derecho Informático de la Facultad de Derecho de la Universidad de Chile, que tiene por objeto difundir en la comunidad jurídica los elementos necesarios para analizar y comprender los alcances y efectos que el desarrollo tecnológico y cultural han producido en la sociedad, especialmente su impacto en la ciencia jurídica.

\author{
EDITOR GENERAL \\ Daniel Álvarez Valenzuela \\ (dalvarez@derecho.uchile.cl) \\ SITIO WEB \\ rchdt.uchile.cl \\ CORREO ELECTRÓNICO \\ rchdt@derecho.uchile.cl \\ LICENCIA DE ESTE ARTÍCULO \\ Creative Commons Atribución Compartir Igual 4.o Internacional
}

\begin{abstract}
La edición de textos, el diseño editorial
y la conversión a formatos electrónicos de este artículo

estuvieron a cargo de Tipográfica

(www.tipografica.io).
\end{abstract}

